\begin{tabular}{|c|l|}
\hline Title & White light channeled imaging polarimeter using broadband polarization gratings \\
\hline Author(s) & Kudenov, Michael W.; Escuti, Michael J.; Dereniak, Eustace L.; Oka, Kazuhiko \\
\hline Citation & $\begin{array}{l}\text { A pplied Optics, 50(15), 2283-2293 } \\
\text { https://doi.org/10.1364/A 0.50.002283 }\end{array}$ \\
\hline Issue Date & 2011-05-20 \\
\hline Doc URL & http://hdl.handle.net/2115/47100 \\
\hline Rights & ○ 2011 Optical Society of A merica \\
\hline Type & article \\
\hline File Information & A 050-15_22832293.pdf \\
\hline
\end{tabular}

Instructions for use 


\title{
White-light channeled imaging polarimeter using broadband polarization gratings
}

\author{
Michael W. Kudenov, ${ }^{1, \star}$ Michael J. Escuti, ${ }^{2}$ Eustace L. Dereniak, ${ }^{1}$ and Kazuhiko Oka ${ }^{3}$ \\ ${ }^{1}$ College of Optical Science, The University of Arizona, 1630 E. University Boulevard, Tucson, Arizona 85721, USA \\ ${ }^{2}$ Department of Electrical and Computer Engineering, North Carolina State University, \\ 2410 Campus Shore Drive, Raleigh, North Carolina 27606, USA \\ ${ }^{3}$ Division of Applied Physics, Hokkaido University, N-13, W-8, Sapporo 060-8628, Japan \\ *Corresponding author: mkudenov@optics.arizona.edu
}

Received 30 November 2010; revised 2 March 2011; accepted 7 March 2011; posted 9 March 2011 (Doc. ID 138651); published 18 May 2011

\begin{abstract}
A white-light snapshot channeled linear imaging (CLI) polarimeter is demonstrated by utilizing polarization gratings (PGs). The CLI polarimeter is capable of measuring the two-dimensional distribution of the linear Stokes polarization parameters by incorporating two identical PGs, in series, along the optical axis. In this configuration, the general optical shearing functionality of a uniaxial crystal-based Savart plate is realized. However, unlike a Savart plate, the diffractive nature of the PGs creates a linear dependence of the shear versus wavelength, thus providing broadband functionality. Consequently, by incorporating the PG-based Savart plate into a Savart plate channeled imaging polarimeter, white-light interference fringes can be generated. This enables polarimetric image data to be acquired at shorter exposure times in daylight conditions, making it more appealing over the quasi-monochromatic channeled imaging polarimeters previously described in the literature. Furthermore, the PG-based device offers significantly more compactness, field of view, optical simplicity, and vibration insensitivity than previously described white-light CLI polarimeters based on Sagnac interferometers. Included in this paper are theoretical descriptions of the linear $\left(S_{0}, S_{1}\right.$, and $\left.S_{2}\right)$ and complete $\left(S_{0}, S_{1}, S_{2}\right.$, and $\left.S_{3}\right)$ channeled Stokes imaging polarimeters. Additionally, descriptions of our calibration procedures and our experimental proof of concept CLI system are provided. These are followed by laboratory and outdoor polarimetric measurements of $S_{0}, S_{1}$, and $S_{2}$. (C) 2011 Optical Society of America

OCIS codes: $\quad 110.3175,300.6190,110.5405$.
\end{abstract}

\section{Introduction}

Imaging polarimetry is used to measure the twodimensional (2D) spatial Stokes parameter distribution of a scene. It is a valuable tool in the characterization of aerosol size distributions, distinguishing man-made targets from background clutter, quality control for evaluating the distribution of stress birefringence, and for evaluating biological tissues in medical imaging [1,2]. The Stokes vector is defined as

0003-6935/11/152283-11\$15.00/0

(C) 2011 Optical Society of America

$$
\mathbf{S}(x, y)=\left[\begin{array}{c}
S_{0}(x, y) \\
S_{1}(x, y) \\
S_{2}(x, y) \\
S_{3}(x, y)
\end{array}\right]=\left[\begin{array}{c}
I_{0}(x, y)+I_{90}(x, y) \\
I_{0}(x, y)-I_{90}(x, y) \\
I_{45}(x, y)-I_{135}(x, y) \\
I_{R}(x, y)-I_{L}(x, y)
\end{array}\right]
$$

where $x, y$ are spatial coordinates in the scene, $S_{0}$ is the total power of the beam, $S_{1}$ denotes preference for linear $0^{\circ}$ over $90^{\circ}, S_{2}$ for linear $45^{\circ}$ over $135^{\circ}$, and $S_{3}$ for right circular over left circular polarization states. By measuring all four elements of $\mathbf{S}(x, y)$, the complete spatial distribution of the polarization state can be determined.

Typically, Stokes parameters are measured by recording four intensity measurements sequentially using different configurations of polarization 
analyzers. By utilizing the instrument's polarimetric response, all Stokes parameters can be calculated by using Mueller matrix calculus. However, timesequential measurements of a rapidly changing scene are susceptible to temporal misregistration. For instance, any motion-induced intensity variations that occur between the time-sequential measurements will appear as a false depolarization signature. Therefore, in order to avoid temporal registration error, all intensity measurements must be recorded in parallel. There are several instruments that can measure complete Stokes vectors or individual Stokes parameters within a single snapshot of a camera(s). These include division of focal plane (DoFP), division of amplitude (DoAM), division of aperture (DoA), and channeled imaging polarimeters (CIPs) [1].

Here, we focus on CIPs [3-5]. In a CIP, interferometrically generated carrier frequencies are amplitude modulated with the spatially dependent $2 \mathrm{D}$ Stokes parameters. Two advantages of a CIP, over the previously listed polarimeters (DoFP, DoAM, and DoA), include its inherent image registration and simple optical implementation. Namely, image registration is inherent since all the Stokes parameters are directly modulated onto coincident interference fringes. Additionally, the optical instrumentation required to incorporate CIP can be augmented onto nearly any pre-existing lens and camera system with minimal effort.

In this paper, we present an instrumentation improvement for CIP. Specifically, we demonstrate a significantly more compact version of our white-light CIP, originally based on a modified Sagnac interferometer [6,7] or an achromatic Fourier transforming lens [8]. By incorporating polarization gratings (PGs) as a diffractive Savart plate, the functional form of the Sagnac interferometer's white-light fringes are preserved [9]. In Section 2, we outline the theoretical basis of the white-light channeled linear imaging (CLI) polarimeter with polarization gratings. Section 3 describes the calibration procedure for the CLI polarimeter, followed by an experimental verification and validation of the system in Section $\underline{4}$. Section $\underline{4}$ also includes details of our outdoor testing. Finally, Appendix A theoretically describes the required system modifications to measure a complete Stokes vector in white light.

\section{Channeled Imaging Polarimetry Using Polarization Gratings}

The basic schematic for the CLI polarimeter is depicted in Fig. 1. It consists of two polarization gratings in series $\left(\mathrm{PG}_{1}\right.$ and $\left.\mathrm{PG}_{2}\right)$, both with a period $\Lambda$ and separated by a distance $t$. PGs in this configuration have been used in laser beam steering [10]. However, as we will demonstrate in this paper, applying them in spectrally and spatially incoherent light enables the generation of white-light interference fringes, analogous to [11]. These two PGs are followed by a linear polarizer (LP) oriented with its transmission axis at $0^{\circ}$ with respect to the $x$ axis.

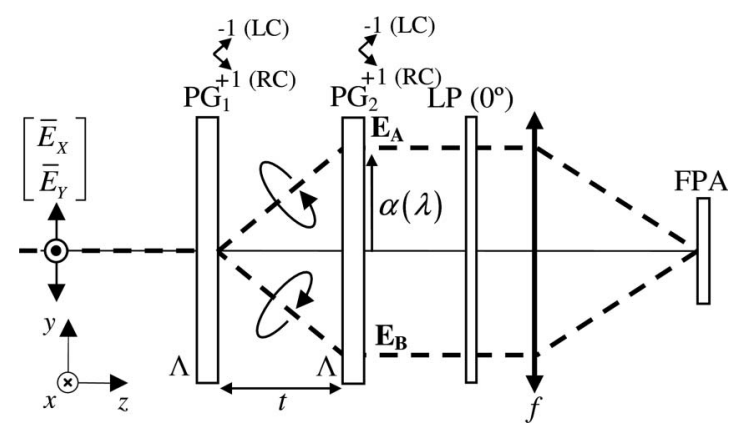

Fig. 1. Schematic of the CLI polarimeter. Right circular (RC) and left circular (LC) polarizations diffract into the -1 st and +1 st diffraction orders, respectively.

An objective lens, with focal length $f$, images collimated light onto the focal plane array (FPA).

The PGs employed here are members of the birefringent diffractive optical elements class [12-14] including spatially periodic birefringence effects, which are, in this case, realized using liquid crystal (LC) materials [14-18]. Individually, they act as a thinfilm beam splitter that is functionally analogous to a Wollaston prism. In both elements, incident light is angularly separated into two forward-propagating, orthogonal polarizations. However, PGs are an embodiment of the Pancharatnam--Berry phase $[19,20]$ operating on circular eigen-polarizations, whereas Wollaston prisms employ the double refraction effect and operate on linearly polarized light. Details of the LC microstructure and holographic fabrication can be found elsewhere [16,17].

The polarization behavior and diffraction efficiency spectra of this PG are notably different than conventional phase or amplitude gratings. While its natural eigen-polarizations are circular (i.e., linearly proportional to $S_{3} / S_{0}$ ), it can be paired [17] with a quarter-wave plate (QWP) in order to separate incident light based on other desired polarizations (i.e., $S_{1} / S_{0}$ or $S_{2} / S_{0}$ ). Light diffracted from the PGs is directed almost entirely into the first $(m= \pm 1)$ or zero $(m=0)$ diffraction orders, where angles are defined by the classical grating equation $\left(\sin \theta_{m}=m \lambda / \Lambda-\right.$ $\sin \theta_{\text {in }}$, where $\Lambda$ is the grating period, $m$ is the grating order, and $\theta_{m}$ and $\theta_{\text {in }}$ are the diffracted and incidence angles, respectively). The diffraction efficiency of a $\mathrm{PG}$ is accurately described in most cases $[14, \underline{17}]$ by

$$
\begin{gathered}
\eta_{ \pm 1}=\left(\frac{1}{2} \mp \frac{S_{3}}{2 S_{0}}\right) K, \\
\eta_{0}=(1-K),
\end{gathered}
$$

where $K$ is a factor determined by the LC structure in the PG.

Our CLI polarimeter incorporates PGs that are capable of high efficiency operation over a broad (white-light) spectrum. The original LC-based PG $[16,17]$ manifests a diffraction efficiency spectrum that is fairly narrow [15], where high first-order efficiency $(>99 \%)$ occurs at wavelengths close to a specified design wavelength $\lambda_{0}$ (within $\Delta \lambda / \lambda_{0} \sim 13 \%$ ). 
Through optimization and refinement of the diffractive structure, a broadband PG [15] enables a larger high efficiency spectral bandwidth $\left(\Delta \lambda / \lambda_{0} \sim 56 \%\right)$, which can cover most of the visible wavelength range. In this case, a good approximation is $K=1$, so that $\eta_{ \pm 1}=1$ and $\eta_{0}=0$ for most visible wavelengths (e.g., $450-750 \mathrm{~nm}$ ).

In our CLI polarimeter, incident light is transmitted by $\mathrm{PG}_{1}$ and initially diffracted into its left and right circularly polarized components, propagating above and below the optical axis, respectively. After transmission through $\mathrm{PG}_{2}$, the two beams $\left(\mathbf{E}_{\mathbf{A}}\right.$ and $\left.\mathbf{E}_{\mathbf{B}}\right)$ are diffracted again to propagate parallel to the optical axis and are now sheared by a distance $\alpha$. The linear polarizer analyzes both beams, thus unifying the polarization state. Imaging both beams onto the FPA combines the two beams and produces interference fringes. To calculate the intensity pat-

$$
\begin{gathered}
\mathbf{E}_{\mathbf{A}}=\mathbf{J}_{-1, \mathrm{LC}} \mathbf{E}_{\mathbf{i n c}}=\frac{1}{2}\left[\begin{array}{c}
\bar{E}_{X}(\xi, \eta-\alpha)-j \bar{E}_{Y}(\xi, \eta-\alpha) \\
j \bar{E}_{X}(\xi, \eta-\alpha)+\bar{E}_{Y}(\xi, \eta-\alpha)
\end{array}\right], \\
\mathbf{E}_{\mathbf{B}}=\mathbf{J}_{+1, \mathrm{RC}} \mathbf{E}_{\text {inc }}=\frac{1}{2}\left[\begin{array}{c}
\bar{E}_{X}(\xi, \eta+\alpha)+j \bar{E}_{Y}(\xi, \eta+\alpha) \\
-j \bar{E}_{X}(\xi, \eta+\alpha)+\bar{E}_{Y}(\xi, \eta+\alpha)
\end{array}\right],
\end{gathered}
$$

where $\alpha$ is the shear, calculated using the paraxial approximation as

$$
\alpha \cong \frac{m \lambda}{\Lambda} t
$$

and $m$ is the diffraction order (being either 1 or -1 ). The total electric field incident on the linear polarizer (LP) is

$$
\begin{aligned}
& \mathbf{E}_{\mathbf{L P}}^{+}=\mathbf{E}_{\mathbf{A}}+\mathbf{E}_{\mathbf{B}}=\frac{1}{2}\left[\begin{array}{c}
\bar{E}_{X}(\xi, \eta+\alpha)+j \bar{E}_{Y}(\xi, \eta+\alpha)+\bar{E}_{X}(\xi, \eta-\alpha)-j \bar{E}_{Y}(\xi, \eta-\alpha) \\
-j \bar{E}_{X}(\xi, \eta+\alpha)+\bar{E}_{Y}(\xi, \eta+\alpha)+j \bar{E}_{X}(\xi, \eta-\alpha)+\bar{E}_{Y}(\xi, \eta-\alpha)
\end{array}\right] . \\
& \mathbf{E}_{\mathbf{L P}}^{-}=\left[\begin{array}{ll}
1 & 0 \\
0 & 0
\end{array}\right] \mathbf{E}_{\mathbf{L P}}^{+}=\frac{1}{2}\left[\begin{array}{c}
\bar{E}_{X}(\xi, \eta+\alpha)+j \bar{E}_{Y}(\xi, \eta+\alpha)+\bar{E}_{X}(\xi, \eta-\alpha)-j \bar{E}_{Y}(\xi, \eta-\alpha) \\
0
\end{array}\right] .
\end{aligned}
$$

Transmission through the linear polarizer, with its

tern on the FPA, assume an arbitrarily polarized electric field is incident on the first polarization grating $\left(\mathrm{PG}_{1}\right)$. The incident field can be expressed as

$$
\mathbf{E}_{\mathbf{i n c}}=\left[\begin{array}{c}
\bar{E}_{X} \\
\bar{E}_{Y}
\end{array}\right]=\left[\begin{array}{l}
E_{X}(\xi, \eta) e^{j \varphi_{x}(\xi, \eta)} \\
E_{Y}(\xi, \eta) e^{j \varphi_{y}(\xi, \eta)}
\end{array}\right]
$$

where $\xi, \eta$ are the angular spectrum components of $x$ and $y$, respectively. The PG's +1 st and -1 st diffraction orders can be modeled as right and left circular polarization analyzers with their Jones matrices expressed as

$$
\begin{aligned}
& \mathbf{J}_{+1, \mathrm{RC}}=\frac{1}{2}\left[\begin{array}{cc}
1 & i \\
-i & 1
\end{array}\right], \\
& \mathbf{J}_{-1, \mathrm{LC}}=\frac{1}{2}\left[\begin{array}{cc}
1 & -i \\
i & 1
\end{array}\right] .
\end{aligned}
$$

After transmission through $\mathrm{PG}_{1}$ and $\mathrm{PG}_{2}$, the $x$ and $y$ polarization components of the electric field, for each of the two beams, are
The objective lens produces a Fourier transformation of the field as

$$
\begin{aligned}
\mathbf{E}_{\mathbf{f}} & =\mathrm{F}\left[\mathbf{E}_{\mathbf{L P}}^{-}\right]_{\xi=\frac{x}{\lambda f}, \eta=\frac{y}{f f}} \\
& =\frac{1}{2}\left[\bar{E}_{X} e^{\frac{2 \pi \pi}{\partial f} \alpha y}+j \bar{E}_{Y} e^{j \frac{2 \pi}{\partial f} \alpha y}+\bar{E}_{X} e^{-j \frac{2 \pi}{\partial f} \alpha y}-j \bar{E}_{Y} e^{-j \frac{2 \pi}{d f} \alpha y}\right],
\end{aligned}
$$

where $\bar{E}_{X}$ and $\bar{E}_{Y}$ are now implicitly dependent upon $x$ and $y$ and $f$ is the focal length of the objective lens. Total electric field intensity can be written as follows:

$$
\begin{aligned}
I= & \left|\mathbf{E}_{\mathbf{f}}\right|^{2} \\
= & \frac{1}{2}\left(\left|\bar{E}_{X}\right|^{2}+\left|\bar{E}_{Y}\right|^{2}\right)+\frac{1}{4}\left(\bar{E}_{X} \bar{E}_{X}^{*}-\bar{E}_{Y} \bar{E}_{Y}^{*}\right) e^{j \frac{2 \pi 2 \alpha \alpha}{\bar{l}} 2 \alpha y} \\
& +\frac{1}{4}\left(\bar{E}_{X} \bar{E}_{X}^{*}-\bar{E}_{Y} \bar{E}_{Y}^{*}\right) e^{-j \frac{2 \pi}{\lambda f} 2 \alpha y} \\
& +j \frac{1}{4}\left(\bar{E}_{X} \bar{E}_{Y}^{*}+\bar{E}_{Y} \bar{E}_{X}^{*}\right) e^{j \frac{j \pi}{\lambda f} 2 \alpha y} \\
& -j \frac{1}{4}\left(\bar{E}_{X} \bar{E}_{Y}^{*}+\bar{E}_{Y} \bar{E}_{X}^{*}\right) e^{-j \frac{2 \pi 2 \alpha y}{\lambda f} .}
\end{aligned}
$$

Simplification using the Stokes parameter definitions [21] yields the final expression for the intensity pattern: 


$$
\begin{aligned}
I(x, y)= & \frac{1}{2}\left[S_{0}(x, y)+S_{1}(x, y) \cos \left(\frac{2 \pi}{\lambda f} 2 \alpha y\right)\right. \\
& \left.+S_{2}(x, y) \sin \left(\frac{2 \pi}{\lambda f} 2 \alpha y\right)\right] .
\end{aligned}
$$

Consequently, the intensity recorded on the FPA contains the amplitude modulated Stokes parameters $S_{0}, S_{1}$, and $S_{2}$. Substitution of the shear into Eq. (14) produces

$$
\begin{aligned}
I(x, y)= & \frac{1}{2}\left[S_{0}(x, y)+S_{1}(x, y) \cos \left(2 \pi \frac{2 m t}{f \Lambda} y\right)\right. \\
& \left.+S_{2}(x, y) \sin \left(2 \pi \frac{2 m t}{f \Lambda} y\right)\right] .
\end{aligned}
$$

From the Eq. (15), the frequency of the interference fringe, or the carrier frequency, denoted by $U$, is

$$
U=\frac{2 m t}{f \Lambda} .
$$

Thus, the linear Stokes parameters are amplitude modulated onto spectrally broadband (white-light) interference fringes. Extending this technique to full Stokes imaging polarimetry is detailed in Appendix A. However, our calibration technique and experimental demonstration focuses on the linear polarimeter due to $\mathrm{PG}$ availability.

\section{CLI Polarimeter Calibration}

The CLI polarimeter is calibrated by applying the reference beam calibration technique $[\underline{4}, \underline{8}]$. First, a for-

parameters are modulated (i.e., convolved) by two shifted $( \pm U)$ delta functions, while the $S_{0}$ Stokes parameter remains unmodulated. These three channels are denoted as $C_{0}\left(S_{0}\right), C_{1}\left(\left(S_{1}-i S_{2}\right) * \delta(\xi, \eta-U)\right)$, and $C_{1}^{*}\left(\left(S_{1}+i S_{2}\right) * \delta(\xi, \eta+U)\right)$. Applying a $2 \mathrm{D}$ filter to two of the three channels $\left(C_{0}\right.$ and $C_{1}$ or $\left.C_{1}^{*}\right)$, followed by an inverse Fourier transformation, enables their content to be isolated from the other components. Inverse Fourier transformation of channels $C_{0}$ and $C_{1}$ produces

$$
\begin{gathered}
C_{0}=\frac{1}{2} S_{0}(x, y) \\
C_{1}=\frac{1}{4}\left(S_{1}(x, y)-i S_{2}(x, y)\right) e^{i 2 \pi U y} .
\end{gathered}
$$

Therefore, the $S_{0}$ Stokes parameter can be extracted directly from Eq. (18), while the $S_{1}$ and $S_{2}$ components are modulated by an exponential phase factor $e^{i 2 \pi U y}$. Isolating this phase factor from the sample data $\left(C_{0, \text { sample }}\right.$ and $\left.C_{1 \text {,sample }}\right)$ is accomplished by comparing it to a previously measured reference polarization state $\left(C_{0, \text { ref }}\right.$ and $\left.C_{1 \text {,ref }}\right)$ containing the known distribution $\left[S_{0, \text { ref }}, S_{1, \text { ref }}, S_{2, \text { ref }}, S_{3, \text { ref }}\right]^{T}$. The sample's Stokes parameters are demodulated by dividing the sample data by the reference data, followed by normalization to the $S_{0}$ Stokes parameter and extraction of the real and imaginary parts:

$$
S_{0}(x, y)=\left|C_{0, \text { sample }}\right|,
$$

$$
\begin{aligned}
& \frac{S_{1}(x, y)}{S_{0}(x, y)}=\mathfrak{R}\left[\frac{C_{1, \text { sample }}}{C_{1, \text { reference }}} \frac{C_{0, \text { reference }}}{C_{0, \text { sample }}}\left(\frac{S_{1, \text { ref }}(x, y)-i S_{2, \text { ref }}(x, y)}{S_{0, \text { ref }}(x, y)}\right)\right], \\
& \frac{S_{2}(x, y)}{S_{0}(x, y)}=\mathfrak{\Im}\left[\frac{C_{1, \text { sample }}}{C_{1, \text { reference }}} \frac{C_{0, \text { reference }}}{C_{0, \text { sample }}}\left(\frac{S_{1, \text { ref }}(x, y)-i S_{2, \text { ref }}(x, y)}{S_{0, \text { ref }}(x, y)}\right)\right] .
\end{aligned}
$$

ward 2D Fourier transformation is performed on the intensity pattern of Eq. (15), producing

$$
\begin{aligned}
I(\xi, \eta)= & F[I(x, y)] \\
= & \frac{1}{2} S_{0}(\xi, \eta)+\frac{1}{4} S_{1}(\xi, \eta) *[\delta(\xi, \eta+U)+\delta(\xi, \eta-U)] \\
& +i \frac{1}{4} S_{2}(\xi, \eta) *[\delta(\xi, \eta+U)-\delta(\xi, \eta-U)],
\end{aligned}
$$

where $\xi$ and $\eta$ are the Fourier transform variables for $x$ and $y$, respectively, and $\delta$ is the Dirac delta function. Equation (17) indicates the presence of three "channels" in the Fourier domain. The $S_{1}$ and $S_{2}$ Stokes
For instance, using reference data created by a linear polarizer, oriented at $0^{\circ}\left[S_{0}, S_{1}, S_{2}, S_{3}\right]^{T}=[1,1,0,0]^{T}$, yields the following reference-beam calibration equations:

$$
\begin{gathered}
S_{0}(x, y)=\left|C_{0, \text { sample }}\right|, \\
\frac{S_{1}(x, y)}{S_{0}(x, y)}=\mathfrak{R}\left[\frac{C_{1, \text { sample }}}{C_{1, \text { reference }}} \frac{C_{0, \text { reference }}}{C_{0, \text { sample }}}\right], \\
\frac{S_{2}(x, y)}{S_{0}(x, y)}=\mathfrak{I}\left[\frac{C_{1, \text { sample }}}{C_{1, \text { reference }}} \frac{C_{0, \text { reference }}}{C_{0, \text { sample }}}\right] .
\end{gathered}
$$




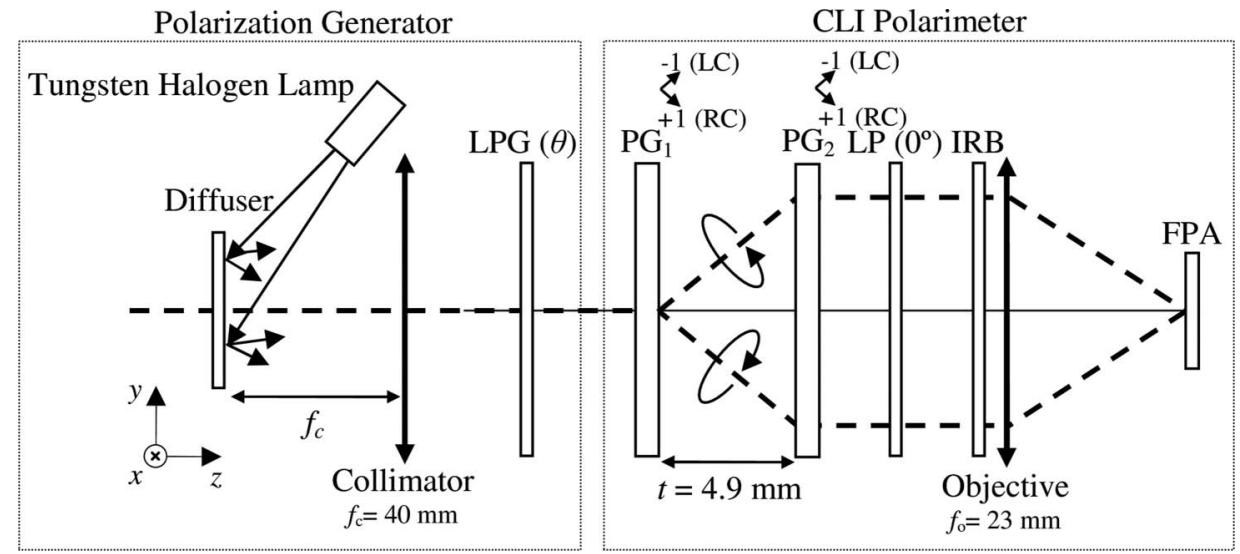

Fig. 2. Experimental setup for establishing the measurement accuracy of the CLI polarimeter in white light.

Equations (23)-(25) are applied to the measured data in order to extract the scene's spatially dependent Stokes parameters.

\section{Experimental Verification of the CLI Polarimeter}

The experimental configuration for establishing the measurement accuracy of the CLI polarimeter in white light with our proof of concept device is illustrated in Fig. 2. It contains a linear polarization generator (LPG) consisting of a tungsten-halogen fiber lamp illuminating a diffuse white ceramic plate. The diffuser is positioned near the focal point of a collimating lens with an effective focal length, $f_{c}$, of $40 \mathrm{~mm}$. Collimated light propagates to a linear polarizer oriented with its transmission axis at $\theta$. This polarization generator produces a uniformly polarized scene for the CLI polarimeter to image. The period of each polarization grating $\left(\mathrm{PG}_{1}\right.$ and $\left.\mathrm{PG}_{2}\right)$ is $\Lambda=$ $7.9 \mu \mathrm{m}$ and the focal length of the objective lens is $f_{o}=23 \mathrm{~mm}$. An infrared blocking filter (IRB) is used in front of the objective lens to limit the spectral passband of the imaged light to $410-750 \mathrm{~nm}$. Last, the FPA is an 8 bit monochrome machine vision camera, containing $640 \times 480$ pixels, placed at the focal point of the objective lens.

\section{A. Polarization Grating Performance}

To assess the performance of the PGs, the zeroth diffraction order's transmission was measured. This provides an approximate metric for how efficiently the PGs are diffracting light into the \pm 1 diffraction orders. Results of the transmission measurements are shown in Fig. 3, demonstrating that the gratings are highly efficient for wavelengths spanning $500-750 \mathrm{~nm}$, but rather inefficient below $475 \mathrm{~nm}$. Consequently, the zero-order light transmitting below $475 \mathrm{~nm}$ will cause error in the calculated Stokes parameters, primarily in the normalization of the measured Stokes parameters to $S_{0}$. Expressing the Stokes parameters in Eq. (15) as spectrally bandintegrated functions yields

$$
S_{n}^{\prime}(x, y)=\int_{\lambda_{1}}^{\lambda_{2}} \operatorname{DE}^{2}(\lambda) S_{n}(x, y, \lambda) \mathrm{d} \lambda
$$

where DE is the diffraction efficiency of one PG for the +1 st or -1 st order, the prime superscript on the Stokes parameters indicate that they have been spectrally band integrated, and the subscript $n=0$, 1 , or 2 indicates the $S_{0}, S_{1}$, or $S_{2}$ Stokes parameter, respectively. Here, it is assumed that both PG's have the same DE versus wavelength. If we choose a spectral region where the DE is not ideal, such that the $\mathrm{DE}<1.0$, then some of the light transmitted through the PGs is not diffracted. This can be introduced to the model [Eq. (15)] as an additional unmodulated zero-order undiffracted offset term $\Delta_{\text {offset }}$ :

$$
\begin{aligned}
I(x, y)= & \frac{1}{2}\left[\Delta_{\text {offset }}(x, y)+S_{0}^{\prime}(x, y)\right. \\
& +S_{1}^{\prime}(x, y) \cos \left(2 \pi \frac{2 m t}{f \Lambda} y\right) \\
& \left.+S_{2}^{\prime}(x, y) \sin \left(2 \pi \frac{2 m t}{f \Lambda} y\right)\right] .
\end{aligned}
$$

Reconstructing via Eqs. (23)-(25) will yield the appropriate absolute results for $\bar{S}_{1}$ and $S_{2}$; however, $S_{0}$ will be erroneous due to the additional offset. Therefore, we introduce the new measured normalized Stokes parameters, denoted by double primes:

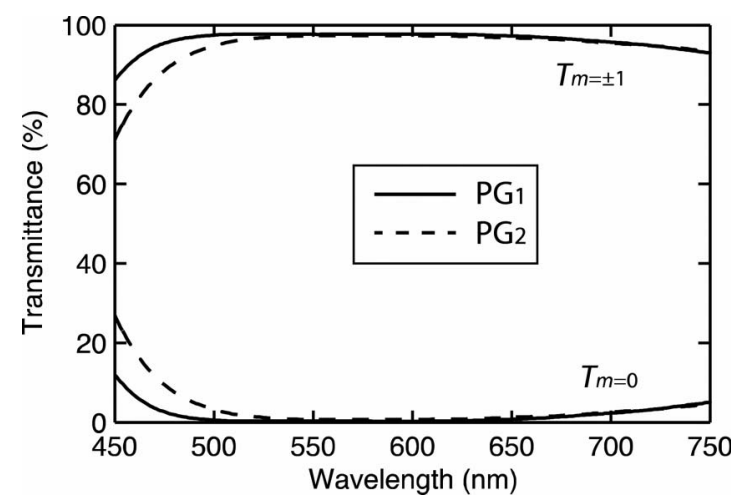

Fig. 3. Measured zero-order and total first-order $\left(T_{ \pm 1}=\right.$ $T_{+1}+T_{-1}$ ) transmission spectra of the PGs. 


$$
\begin{aligned}
& S_{0}^{\prime \prime}(x, y)=S_{0}^{\prime}(x, y)+\Delta_{\text {offset }}(x, y), \\
& \frac{S_{n}^{\prime \prime}(x, y)}{S_{0}^{\prime \prime}(x, y)}=\frac{S_{n}^{\prime}(x, y)}{S_{0}^{\prime}(x, y)+\Delta_{\text {offset }}(x, y)},
\end{aligned}
$$

where the subscript $n=1$ or 2 indicates the $S_{1}$ or $S_{2}$ Stokes parameter, respectively. Consequently, error is induced into the $S_{1}$ and $S_{2}$ Stokes parameters from the normalization to the effectively larger $S_{0}$ component $\left[S_{0}^{\prime}(x, y)+\Delta_{\text {offset }}(x, y)\right]$. While error due to this zero-order light leakage was observed in our outdoor tests, it was negligible in our laboratory characterization because our $S_{0}$ reference and sample illumination levels were constant. Ultimately, we anticipate that PGs with a zero-order light transmission of less than $3 \%$ over the passband would enable better accuracy regardless of the $S_{0}$ illumination level. For our proof of concept experiments, we aim at demonstrating the CLI polarimeter's ability to generate white-light polarization-induced interference fringes.

\section{B. Calibration Verification}

In order to verify the mathematical relationship established by Eq. (15), in addition to the calibration accuracy defined at a constant illumination condition, reference data were taken with the LPG. The LPG was rotated for angles $\theta$ between $0^{\circ}$ and $180^{\circ}$ in $10^{\circ}$ increments. After reconstruction, the central portion of the field of view was averaged over a $100 \times$ 100 pixel area to obtain an average value for the measured polarization state. Images of the whitelight interference fringes from this $100 \times 100$ pixel area are depicted in Fig. $\underline{4}$ for $\theta$ equal to $0^{\circ}, 50^{\circ}$, and $90^{\circ}$.

Note that the phase of the sinusoidal fringes changes while the amplitude remains constant for varying linear polarizer orientations. This phase change is directly related to Eq. (15), and indicates the varying proportions of $S_{1}$ to $\overline{S_{2}}$ as the LPG is rotated. Meanwhile, the amplitude remains constant because the degree of linear polarization (DOLP = $\left.\sqrt{S_{1}^{2}+S_{2}^{2}} / S_{0}\right)$ from the LPG is constant $(\sim 1)$.

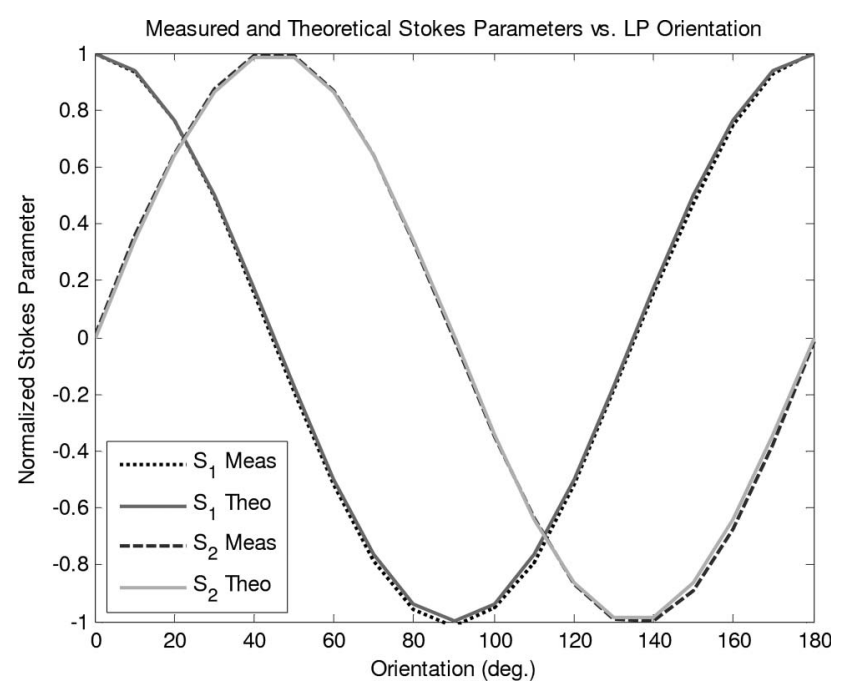

Fig. 5. Measured and theoretical results of the polarimetric reconstructions.

Plotting the measured $S_{1}$ and $S_{2}$ Stokes parameters versus $\theta$ and comparing them to the theoretical values yields the results depicted in Fig. 5 . The calculated RMS error for both curves is approximately $1.6 \%$. This implies that the amplitude modulation of Eq. (15) accurately follows the incident Stokes parameter variation.

\section{Outdoor Measurements}

The snapshot imaging capability of the CLI polarimeter was also assessed outdoors on moving targets. For outdoor scenes, the absolute accuracy of the Stokes parameters for varying illumination levels is not well established, again due to the zero-order diffraction efficiency leakage discussed previously in Subsection 4.A. Consequently, our outdoor results are provided here to demonstrate the snapshot imaging and reconstruction capabilities in full sunlight.

The optical configuration for these tests is depicted in Fig. 6. Included is a 1:1 afocal telescope consisting of two $\overline{5} 0 \mathrm{~mm}$ focal length lenses operating at a focal number of $f / 1.8$. These optics enable defocus to be introduced into the image of the scene, while simultaneously maintaining focus on the fringes that are localized at infinity. Defocus is used to band limit the

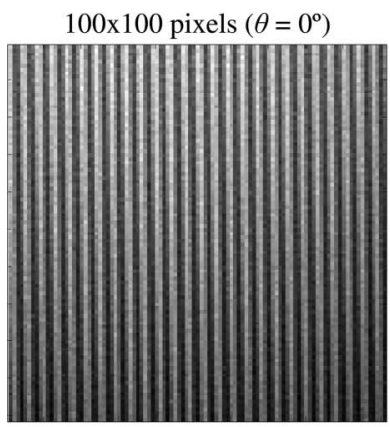

(a)

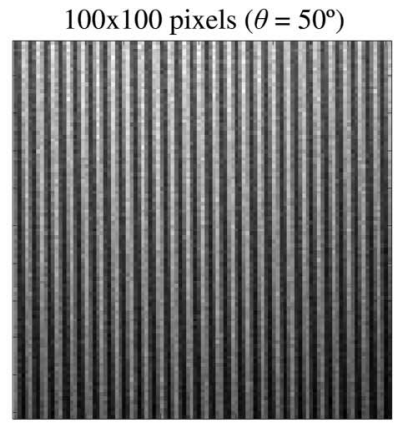

(b)

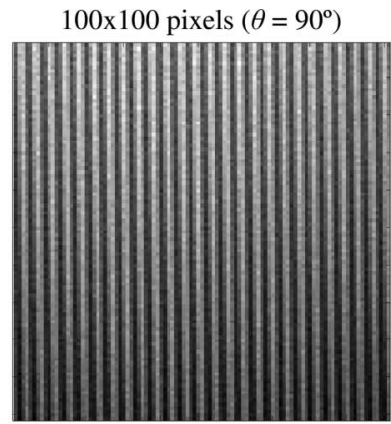

(c)

Fig. 4. White-light interference fringes generated in the central $100 \times 100$ pixels on the focal plane array for generating polarizer orientations of (a) $\theta=0^{\circ}$, (b) $\theta=50^{\circ}$, and (c) $\theta=90^{\circ}$. 


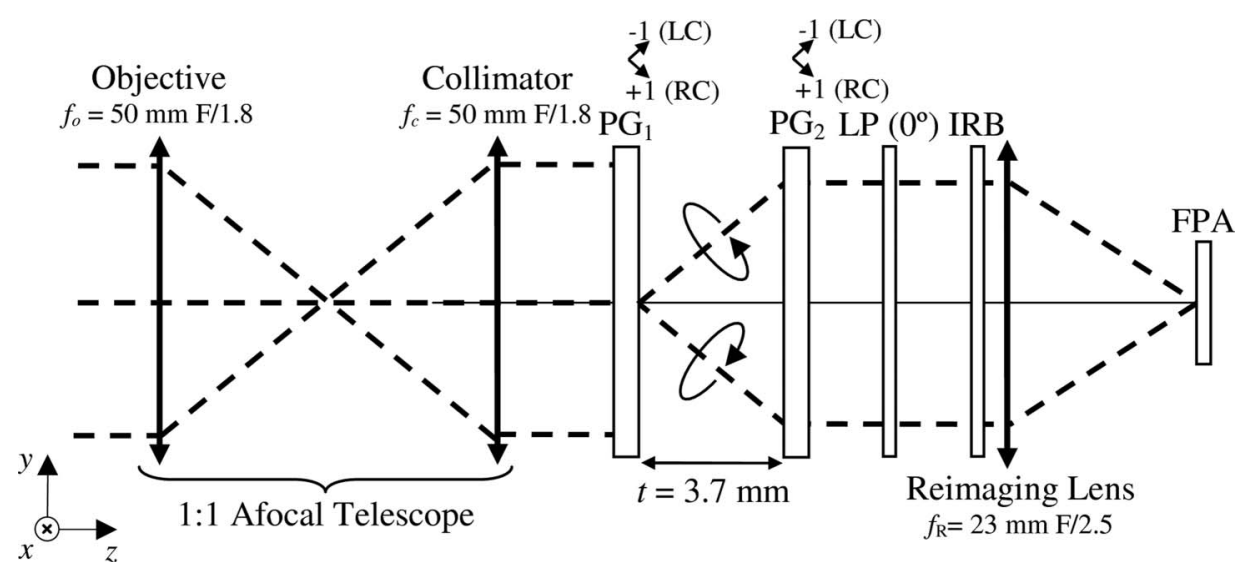

Fig. 6. Experimental setup for viewing outdoor targets with the CLI polarimeter. An afocal telescope is included to allow the scene to be defocused while maintaining focus on the interference fringes.

spatial frequency content of the scene, thereby reducing aliasing artifacts in the reconstructed Stokes parameters [8,22]. A raw image of a moving vehicle, captured with the CLI polarimeter pictured in Fig. 7 , is depicted in Fig. 8. The image was taken on a clear and sunny afternoon with an exposure speed of approximately $1 / 1200$ th of a second with a reimaging lens focal number of $f / 2.5$. Reference data, taken of a linear polarizer oriented at $0^{\circ}$ in front of a diffuser, was measured shortly after the vehicle was imaged. The diffuser was illuminated by sunlight.

The polarization data was extracted by taking a fast Fourier transformation of the raw data, followed by filtration, an inverse Fourier transformation, and calibration by application of Eqs. (23)-(25). Similarly to [22], we have observed reconstruction artifacts due to aliasing. Consequently, the reconstructed data were also processed with an aliasing reduction filter that reduces noise due to aliasing artifacts. This produced the data depicted in Fig. $\underline{9}$, where the DOLP is

$$
\operatorname{DOLP}(x, y)=\frac{\sqrt{S_{1}^{2}(x, y)+S_{2}^{2}(x, y)}}{S_{0}(x, y)} .
$$

The orientation of the linearly polarized light $\left(\theta_{L}\right)$ can be extracted from the measured Stokes parameters using the formula

$$
\theta_{L}(x, y)=\frac{1}{2} \tan ^{-1}\left(\frac{S_{2}(x, y)}{S_{1}(x, y)}\right) .
$$

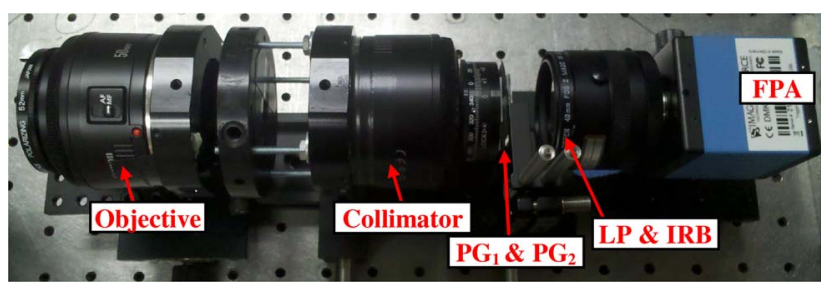

Fig. 7. (Color online) Photo of the CLI polarimeter on the bench top. This configuration of the polarimeter is optimized for viewing scenes outdoors.
By incorporating the color fusion method, this orientation information can be superimposed onto the DOLP and intensity $\left(S_{0}\right)$ information [23]. In color fusion, a hue (pixel color), saturation (amount of color within the pixel), and value (pixel brightness) colormapping is used. This hue-saturation-value (HSV) color map is mapped directly into the linear polarization's orientation (hue), DOLP (saturation), and intensity $S_{0}$ (value). Images generated with this scheme provide a qualitative assessment of the polarimetric and intensity information. The color fusion image of Fig. 10 was generated from the data portrayed in Fig. 9, along with the orientation information calculated from Eq. (31).

\section{Conclusion}

A compact version of a white-light CLI polarimeter was demonstrated. By incorporating two polarization gratings in series, the same functionality of the original Sagnac-interferometer-based implementation, based on [6], is maintained. A theoretical

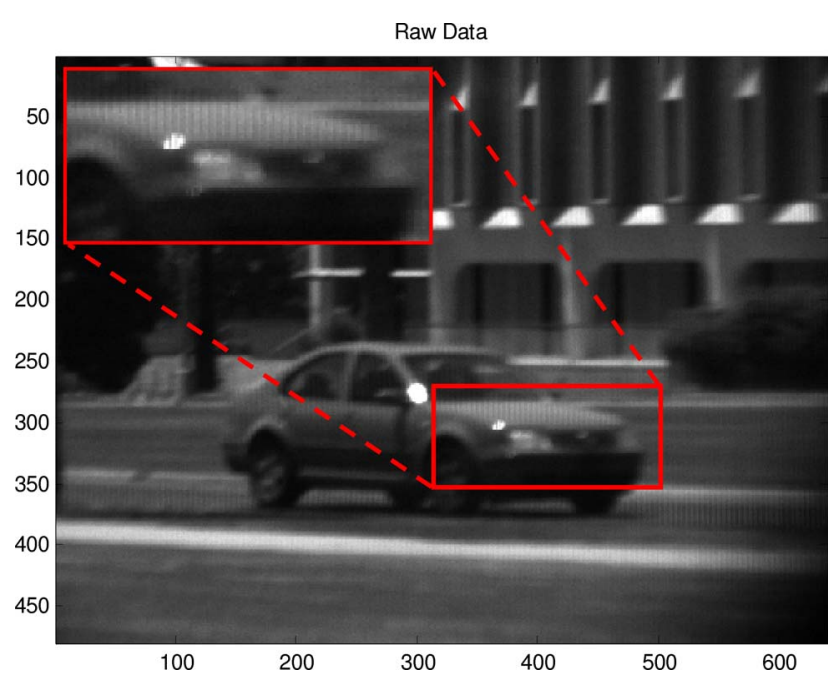

Fig. 8. (Color online) Raw image of a moving vehicle. Interference fringes are located in areas of the scene that are linearly polarized. These fringes are particularly evident in the hood of the vehicle. 


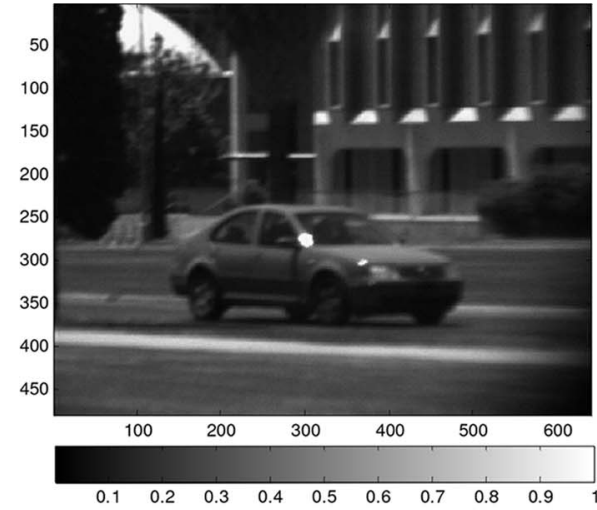

(a)

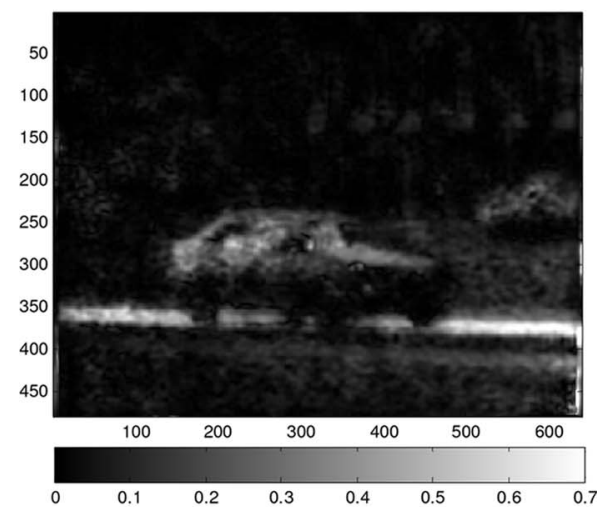

(b)

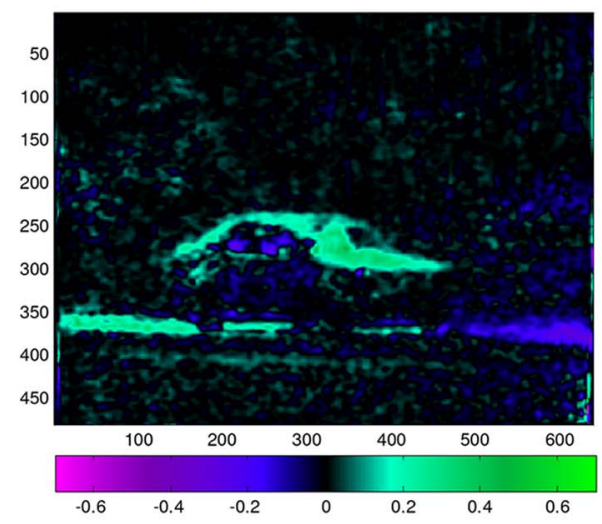

(c)

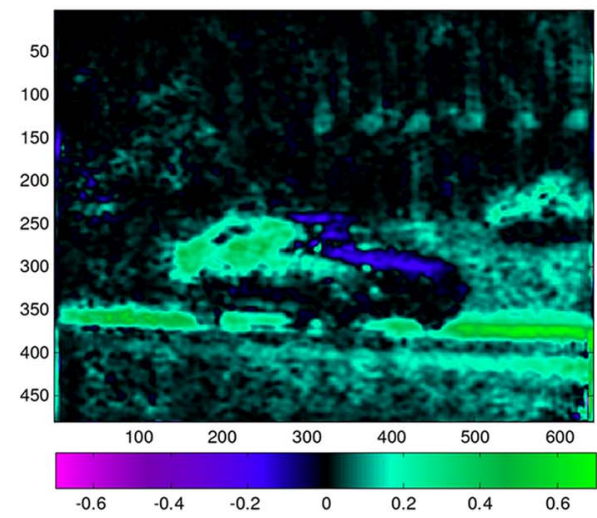

(d)

Fig. 9. (Color online) Processed polarization data of the vehicle, calculated from the raw data in Fig. 8. (a) $S_{0}$, (b) DOLP, (c) $S_{1} / S_{0}$, and (d) $S_{2} / S_{0}$.

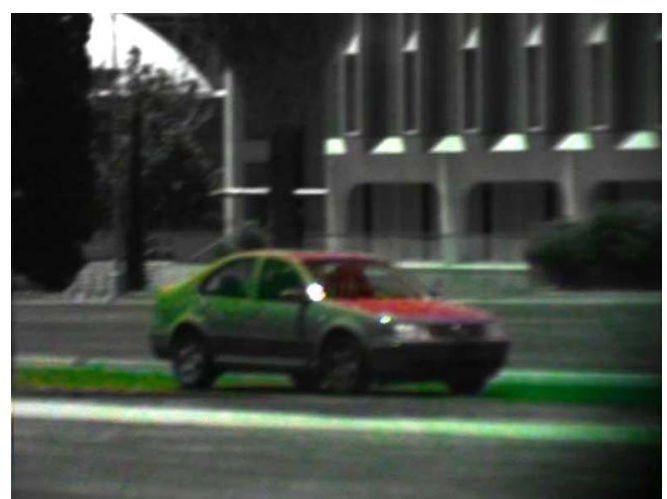

Fig. 10. (Color online) Color fusion image of the vehicle, generated from the spectrally broadband polarization data. Magenta and green represent linearly polarized light with $\theta_{L} \sim-10^{\circ}$ and $45^{\circ}$, respectively. Full saturation corresponds to a DOLP of 0.4 .

model of the linear Stokes polarimeter was provided. Furthermore, raw data from the linear polarimeter proof of concept were reconstructed for the linear polarization states corresponding to the $S_{0}, S_{1}$, and $S_{2}$ Stokes parameters. For our laboratory characterization, measurements revealed a $1.6 \%$ RMS error in the normalized $S_{1}$ and $S_{2}$ Stokes parameters under uniform white-light illumination. We have demonstrated a compact CIP using PGs, which can measure 2D spatial Stokes parameter distributions in whitelight outdoor conditions.

\section{Appendix A: Full Stokes Polarimetry}

The CLI polarimeter can be considered as a subset of the Savart plate Stokes imaging polarimeter originally described in [4]. By replacing each Savart plate with two PGs, a white-light Stokes imaging polarimeter capable of measuring $S_{0}, S_{1}, S_{2}$, and $S_{3}$ can be realized. The optical layout for this scheme is depicted schematically in Fig. 11. Light transmitted by $\mathrm{PG}_{1}$ and $\mathrm{PG}_{2}$ is sheared along the $y$ axis by a distance $\alpha$. The two circularly polarized beams are converted into linearly polarized light after propagation through a QWP. Transmission through $\mathrm{PG}_{3}$ and $\mathrm{PG}_{4}$ shears each of the two beams along the $x$ axis by a distance $\beta$. Propagation of the four circularly polarized beams through another QWP, linear polarizer, and objective lens generates white-light polarization interference fringes at the FPA. Propagation of a single polarized ray through the PGs and QWPs is depicted in three dimensions per Fig. 12.

For the purposes of the following derivation, the $\mathrm{PG}_{1}$ to $\mathrm{PG}_{2}$ separation $\left(t_{1}\right)$ is equal to the $\mathrm{PG}_{3}$ to $\mathrm{PG}_{4}$ separation $\left(t_{2}\right)$, such that $t_{1}=t_{2}=t$. Furthermore, all four PGs have identical grating periods $\Lambda$. The incident arbitrarily polarized electric field is defined as

$$
\mathbf{E}_{\text {inc }}=\left[\begin{array}{c}
\bar{E}_{X} \\
\bar{E}_{Y}
\end{array}\right]=\left[\begin{array}{l}
E_{X}(\xi, \eta) e^{j \phi_{x}(\xi, \eta)} \\
E_{Y}(\xi, \eta) e^{j \phi_{y}(\xi, \eta)}
\end{array}\right] .
$$

After transmission through $\mathrm{PG}_{1}$ and $\mathrm{PG}_{2}$, the $x$ and $y$ components of the electric field for $\mathbf{E}_{\mathbf{A}}$ and $\mathbf{E}_{\mathbf{B}}$ are 


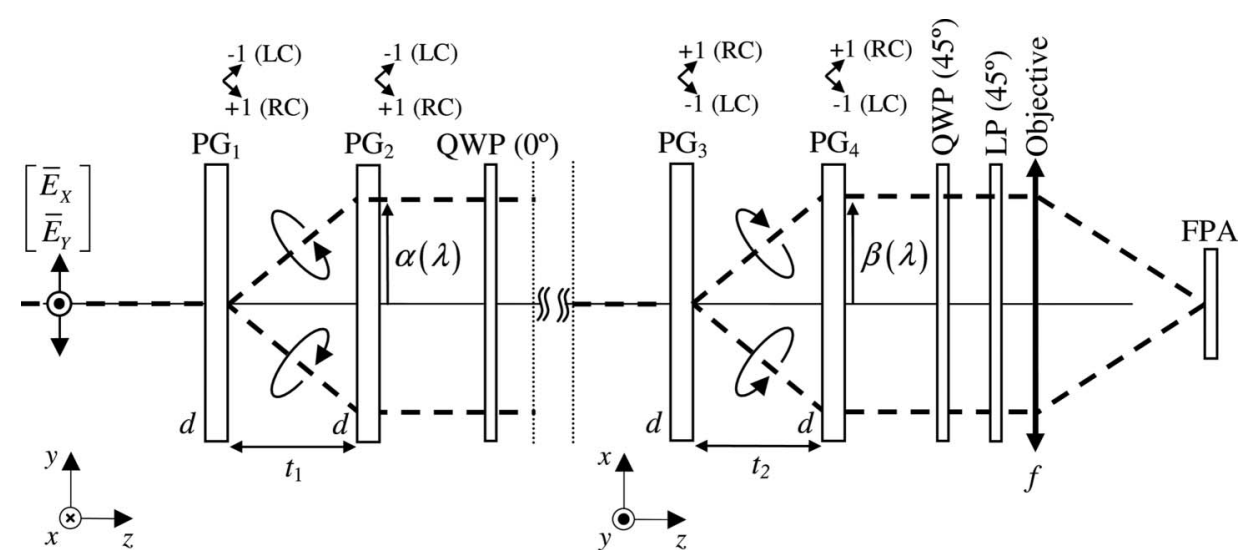

Fig. 11. Schematic of the full imaging Stokes polarimeter. Note that $\mathrm{PG}_{1}$ and $\mathrm{PG}_{2}$ diffract in the $y-z$ plane, while $\mathrm{PG}_{3}$ and $\mathrm{PG}_{4}$ diffract in the $x-z$ plane. The coordinate system has been rotated to portray this clearly in two dimensions.

identical to Eqs. (7) and (8). Propagation through the QWP, oriented with its fast axis at $0^{\circ}$, yields

$$
\mathbf{E}_{\mathbf{A}}^{\prime}=\left[\begin{array}{cc}
1 & 0 \\
0 & -j
\end{array}\right] \mathbf{E}_{\mathbf{A}}=\frac{1}{2}\left[\begin{array}{l}
\bar{E}_{X}(\xi, \eta-\alpha)-j \bar{E}_{Y}(\xi, \eta-\alpha) \\
\bar{E}_{X}(\xi, \eta-\alpha)-j \bar{E}_{Y}(\xi, \eta-\alpha)
\end{array}\right]
$$

$$
\mathbf{E}_{\mathbf{B}}^{\prime}=\left[\begin{array}{cc}
1 & 0 \\
0 & -j
\end{array}\right] \mathbf{E}_{\mathbf{B}}=\frac{1}{2}\left[\begin{array}{c}
\bar{E}_{X}(\xi, \eta+\alpha)+j \bar{E}_{Y}(\xi, \eta+\alpha) \\
-\bar{E}_{X}(\xi, \eta+\alpha)-j \bar{E}_{Y}(\xi, \eta+\alpha)
\end{array}\right]
$$

Propagation of $\mathbf{E}_{\mathbf{A}}^{\prime}$ and $\mathbf{E}_{\mathbf{B}}^{\prime}$ through $\mathrm{PG}_{3}$ and $\mathrm{PG}_{4}$ yields four beams, labeled $\mathbf{E}_{\mathbf{C}}, \mathbf{E}_{\mathbf{D}}, \mathbf{E}_{\mathbf{E}}$, and $\mathbf{E}_{\mathbf{F}}$ in Fig. 12 . These four transmitted fields are expressed by

$$
\begin{aligned}
\mathbf{E}_{\mathbf{C}}(\xi+\alpha, \eta-\alpha) & =\mathbf{J}_{-1, \mathrm{LC}} \mathbf{E}_{\mathbf{A}}^{\prime}(\xi, \eta-\alpha) \\
& =\frac{1}{4}\left[\begin{array}{c}
\left(\bar{E}_{X}-\bar{E}_{Y}\right)-j\left(\bar{E}_{X}+\bar{E}_{Y}\right) \\
\left(\bar{E}_{X}+\bar{E}_{Y}\right)+j\left(\bar{E}_{X}-\bar{E}_{Y}\right)
\end{array}\right],
\end{aligned}
$$

$$
\begin{aligned}
\mathbf{E}_{\mathbf{D}}(\xi-\alpha, \eta-\alpha) & =\mathbf{J}_{+1, \mathrm{RC}} \mathbf{E}_{\mathbf{A}}^{\prime}(\xi, \eta-\alpha) \\
& =\frac{1}{4}\left[\begin{array}{c}
\left(\bar{E}_{X}+\bar{E}_{Y}\right)+j\left(\bar{E}_{X}-\bar{E}_{Y}\right) \\
\left(\bar{E}_{X}-\bar{E}_{Y}\right)-j\left(\bar{E}_{X}+\bar{E}_{Y}\right)
\end{array}\right], \\
\mathbf{E}_{\mathbf{E}}(\xi+\alpha, \eta+\alpha) & =\mathbf{J}_{-1, \mathrm{LC}} \mathbf{E}_{\mathbf{B}}^{\prime}(\xi, \eta+\alpha) \\
& =\frac{1}{4}\left[\begin{array}{c}
\left(\bar{E}_{X}-\bar{E}_{Y}\right)+j\left(\bar{E}_{X}+\bar{E}_{Y}\right) \\
-\left(\bar{E}_{X}+\bar{E}_{Y}\right)+j\left(\bar{E}_{X}-\bar{E}_{Y}\right)
\end{array}\right],
\end{aligned}
$$

$$
\begin{aligned}
\mathbf{E}_{\mathbf{F}}(\xi-\alpha, \eta+\alpha) & =\mathbf{J}_{+1, \mathrm{RC}} \mathbf{E}_{\mathbf{B}}^{\prime}(\xi, \eta+\alpha) \\
& =\frac{1}{4}\left[\begin{array}{c}
\left(\bar{E}_{X}+\bar{E}_{Y}\right)-j\left(\bar{E}_{X}-\bar{E}_{Y}\right) \\
-\left(\bar{E}_{X}-\bar{E}_{Y}\right)-j\left(\bar{E}_{X}+\bar{E}_{Y}\right)
\end{array}\right],
\end{aligned}
$$

where $\bar{E}_{X}$ and $\bar{E}_{Y}$ are implicitly dependent on $\xi, \eta$, and $\alpha$. Transmission through the last QWP, with its fast axis oriented at $45^{\circ}$, rotates the circular polarization states of $\mathbf{E}_{\mathbf{C}}, \mathbf{E}_{\mathbf{D}}, \mathbf{E}_{\mathbf{E}}$, and $\mathbf{E}_{\mathrm{F}}$ into vertical and horizontal linear polarizations. Propagation of these beams

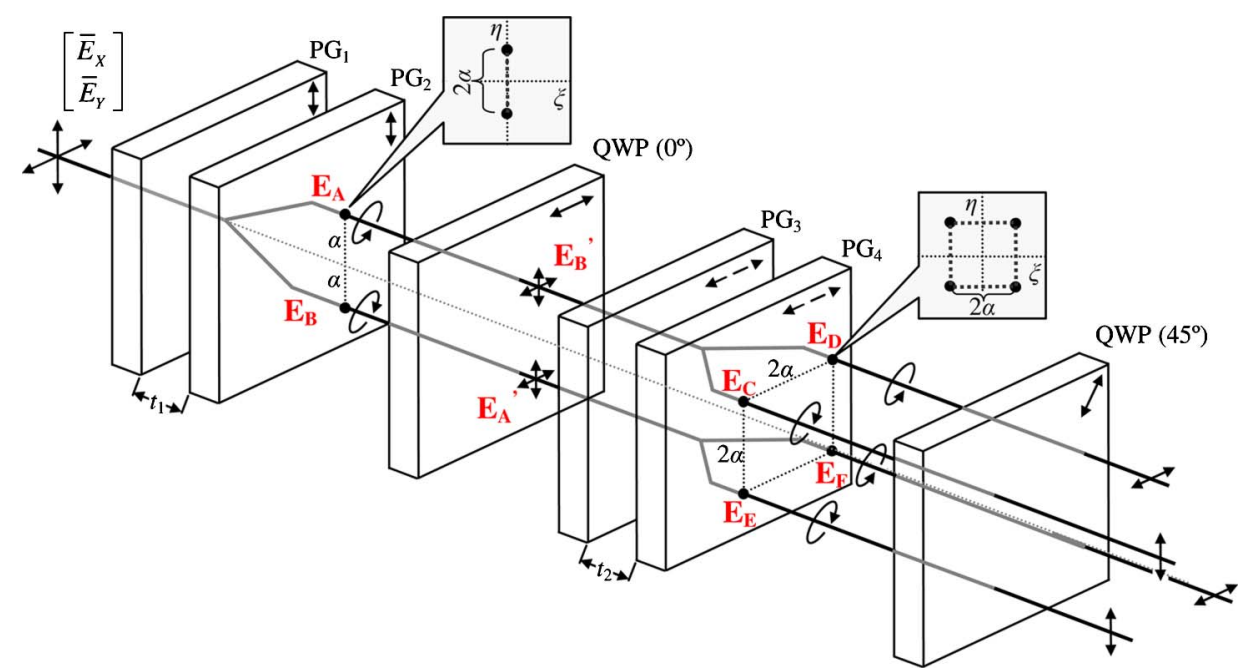

Fig. 12. (Color online) Three-dimensional schematic of the polarization gratings $\mathrm{PG}_{1}$ through $\mathrm{PG}_{4}$, in addition to the two QWPs. 
through the analyzing linear polarizer unifies them into a $45^{\circ}$ linear polarization state. The complete electric field's $x$ and $y$ components incident on the lens is

$$
\begin{aligned}
\mathbf{E}_{\mathbf{X}}^{\mathbf{L}}= & \mathbf{E}_{\mathbf{Y}}^{\mathbf{L}} \\
= & \frac{1}{4}\left(\bar{E}_{X}(\xi+\alpha, \eta-\alpha)-j \bar{E}_{Y}(\xi+\alpha, \eta-\alpha)\right) \\
& +\left(\bar{E}_{X}(\xi-\alpha, \eta-\alpha)-j \bar{E}_{Y}(\xi-\alpha, \eta-\alpha)\right) \\
& +\left(j \bar{E}_{X}(\xi+\alpha, \eta+\alpha)-\bar{E}_{Y}(\xi+\alpha, \eta+\alpha)\right) \\
& +\left(-j \bar{E}_{X}(\xi-\alpha, \eta+\alpha)+\bar{E}_{Y}(\xi-\alpha, \eta+\alpha)\right) .
\end{aligned}
$$

The objective lens produces a Fourier transformation of the field. Performing this on the $\mathbf{E}_{\mathbf{X}}^{\mathbf{L}}$ component yields

$$
\begin{aligned}
\mathbf{E}_{\mathbf{L}}= & F\left[\mathbf{E}_{\mathbf{X}}^{\mathbf{L}}\right]_{\xi=\frac{x}{\lambda f}, \eta=\frac{y}{\lambda f}} \\
= & \frac{1}{4}\left(\bar{E}_{X}-j \bar{E}_{Y}\right) e^{j \frac{2 \pi}{\lambda f} \alpha(x-y)}+\left(-j \bar{E}_{X}+\bar{E}_{Y}\right) e^{-j \frac{2 \pi}{\lambda f} \alpha(x-y)} \\
& +\left(j \bar{E}_{X}-\bar{E}_{Y}\right) e^{j \frac{2 \pi}{\lambda f} \alpha(x+y)}+\left(\bar{E}_{X}-j \bar{E}_{Y}\right) e^{-j \frac{2 \pi}{\lambda f} \alpha(x+y)},
\end{aligned}
$$

where $\bar{E}_{X}$ and $\bar{E}_{Y}$ are implicitly dependent on $x$ and $y$, $f$ is the focal length of the objective lens, and $\lambda$ is the wavelength of the incident illumination. The intensity is calculated by taking the absolute value squared of $\mathbf{E}_{\mathbf{L}}$. Simplifying the expression with the Stokes parameter definitions, combining terms into cosines and sines, and substituting the shear $\alpha$ from Eq. (9), produces the final intensity pattern on the FPA:

$$
\begin{aligned}
I(x, y)= & \frac{1}{2} S_{0}(x, y)+\frac{1}{2} S_{3}(x, y) \cos \left(2 \pi \frac{2 m t}{f \Lambda} x\right) \\
& +\frac{1}{4} S_{2}(x, y)\left[\cos \left(2 \pi \frac{2 m t}{f \Lambda}(x-y)\right)\right. \\
& \left.-\cos \left(2 \pi \frac{2 m t}{f \Lambda}(x+y)\right)\right] \\
& +\frac{1}{4} S_{1}(x, y)\left[\sin \left(2 \pi \frac{2 m t}{f \Lambda}(x-y)\right)\right. \\
& \left.+\sin \left(2 \pi \frac{2 m t}{f \Lambda}(x+y)\right)\right]
\end{aligned}
$$

This configuration enables the measurement of all four Stokes parameters by isolating the various white-light spatial carrier frequencies $U_{1}$ and $U_{2}$, defined as

$$
\begin{gathered}
U_{1}=2 \frac{m t}{f \Lambda}, \\
U_{2}=2 \sqrt{2} \frac{m t}{f \Lambda} .
\end{gathered}
$$

For the purposes of this paper, only the linear polarimeter implementation was experimentally verified due to the availability of the polarization gratings.

M. J. Escuti acknowledges support from the National Science Foundation (NSF, grant ECCS0955127).

\section{References}

1. J. S. Tyo, D. L. Goldstein, D. B. Chenault, and J. A. Shaw, "Review of passive imaging polarimetry for remote sensing applications," Appl. Opt. 45, 5453-5469 (2006).

2. E. R. Cochran and C. Ai, "Interferometric stress birefringence measurement," Appl. Opt. 31, 6702-6706 (1992).

3. K. Oka and T. Kaneko, "Compact complete imaging polarimeter using birefringent wedge prisms," Opt. Express 11, 1510-1519 (2003).

4. K. Oka and N. Saito, "Snapshot complete imaging polarimeter using Savart plates," Proc. SPIE 6295, 629508 (2006).

5. J. VanDelden, "Interferometric polarization interrogating filter assembly and method," U.S. patent 6,674,532 B2 (6 January 2004).

6. M. W. Kudenov, M. E. L. Jungwirth, E. L. Dereniak, and G. R. Gerhart, "White light Sagnac interferometer for snapshot linear polarimetric imaging," Opt. Express 17, 22520-22534 (2009).

7. M. Mujat, E. Baleine, and A. Dogariu, "Interferometric imaging polarimeter," J. Opt. Soc. Am. A 21, 2244-2249 (2004).

8. M. W. Kudenov, L. Pezzaniti, E. L. Dereniak, and G. R. Gerhart, "Prismatic imaging polarimeter calibration for the infrared spectral region," Opt. Express 16, 13720-13737 (2008).

9. K. Oka, R. Suda, M. Ohnuki, D. Miller, and E. L. Dereniak, "Snapshot imaging polarimeter for polychromatic light using Savart plates and diffractive lenses," in Frontiers in Optics, OSA Technical Digest (CD) (Optical Society of America, 2009), paper FThF4.

10. C. Oh, J. Kim, J. F. Muth, S. Serati, and M. J. Escuti, "High-throughput, continuous beam steering using rotating polarization gratings," IEEE Photon. Technol. Lett. 22, 200-202 (2010).

11. J. C. Wyant, "OTF measurements with a white light source: an interferometric technique," Appl. Opt. 14, 1613-1615 (1975).

12. L. Nikolova and T. Todorov, "Diffraction efficiency and selectivity of polarization holographic recording," Opt. Acta 31, 579-588 (1984).

13. J. Tervo and J. Turunen, "Paraxial-domain diffractive elements with $100 \%$ efficiency based on polarization gratings," Opt. Lett. 25, 785-786 (2000).

14. C. Oh and M. J. Escuti, "Numerical analysis of polarization gratings using the finite-difference time-domain method," Phys. Rev. A 76, 043815 (2007).

15. C. Oh and M. J. Escuti, "Achromatic diffraction from polarization gratings with high efficiency," Opt. Lett. 33, 2287-2289 (2008).

16. G. P. Crawford, J. N. Eakin, M. D. Radcliffe, A. C. Jones, and R. A. Pelcovits, "Liquid-crystal diffraction gratings using polarization holography alignment techniques," J. Appl. Phys. 98, $123102(2005)$.

17. M. J. Escuti, C. Oh, C. Sanchez, C. W. M. Bastiaansen, and D. J. Broer, "Simplified spectropolarimetry using reactive mesogen polarization gratings," Proc. SPIE 6302, 630207 (2006).

18. R. K. Komanduri, W. M. Jones, C. Oh, and M. J. Escuti, "Polarization-independent modulation for projection displays 
using small-period LC polarization gratings,” J. Soc. Inf. Disp. 15, 589-594 (2007).

19. S. Pancharatnam, "Generalized theory of interference, and its applications. Part I. Coherent pencils," Proc. Indian Acad. Sci. A 44, 247-262 (1956).

20. M. V. Berry, "The adiabatic phase and Pancharatnam's phase for polarized light," J. Mod. Opt. 34, 1401-1407 (1987).
21. D. Goldstein, Polarized Light (Marcel Dekker, 2003).

22. J. Craven and M. W. Kudenov, "False signature reduction in channeled spectropolarimetry," Opt. Eng. 49, 053602 (2010).

23. J. S. Tyo, E. N. Pugh, and N. Engheta, "Colorimetric representations for use with polarization-difference imaging of objects in scattering media," J. Opt. Soc. Am. A 15, 367-374 (1998). 